\title{
Evaluation of biodiesel production from babassu oil and ethanol applying alkaline transesterification under ultrasonic technology
}

\author{
Paiva, E. J. M., Silva, M. L. C. P., Castro, H. F., Barboza, J. C. S., Giordani, D. S. \\ School of Engineering of Lorena - University of São Paulo, Lorena, Brazil \\ *Corresponding author. Tel: +55 12 31595142, Fax: +55 12 31533224, E-mail: giordani@dequi.eel.usp.br
}

\begin{abstract}
Babassu oil is a clear light yellow vegetable oil extracted from the seeds of the babassu palm (Attalea speciosa), which grows in most areas of South America. It is about $70 \%$ lipids, with $50 \%$ of lauric composition. Brazil is the world's second largest producer of ethanol and the world's largest exporter; the advantages of ethanol are concerned to its renewable origin and low toxicity. In this work ethyl esters of babassu oil were synthesized by alkaline catalysis in homogeneous medium. The experimental design was used as a tool for optimization of the transesterification reaction and also in identifying key factors influencing the conversion into ethyl esters. The transesterification reactions were performed using two methods - the traditional mechanical agitation and agitation promoted by ultrasound waves. The nuclear magnetic resonance spectroscopy was used to quantify the conversion of all reactions of transesterification. According to the model obtained by the experimental design for mechanical agitation, conversions above $99 \%$ are obtained when the stoichiometric ratio is set at $6: 1$, with $1.0 \% \mathrm{KOH}$, under stirring at $400 \mathrm{rpm}$, in $60 \mathrm{~min}$. Alkaline transesterification assisted by ultrasound waves produced the best results with respect to time of reaction and phase separation of glycerin and ethyl esters. The experimental model showed that conversions above $99 \%$ can be obtained in 10 min after adjusting the other independent variables.
\end{abstract}

Keywords: Biodiesel, Babassu oil, Transesterification, Ethanol, Ultrasound.

\section{Introduction}

The vegetable oil transesterification yields biodiesel as the main product. However, the final mixture is composed of free glycerol, alcohol, catalyst and unreacted mono-, di- and triglycerides [1]. These contaminants can lead to environmental and operational problems. Achieving high conversions in mono-alkyl esters, to ensure the removal of free glycerin, catalyst, alcohol and fatty acids in biodiesel are critical issues to the quality control and is one of the main challenges to be overcome to make feasible the industrial production of these fuels. In another approach, the fatty acid composition of vegetable oils is a significant factor influencing the performance of biofuels, carbon chains with a high number of unsaturations are more susceptible to oxidation as well as they have better performance with low temperatures, in contrast, saturated chains are desirable [2,3], especially due to the higher oxidation resistance and improved cetane number, but its use in cold climates is conditioned to its cloud point.

Among all feasible vegetable oil to cultivation and to oil extraction, this study employed the babassu, a generic name given to palm oil belonging to the Palmae family and members of the genera Orbignya and Attalea. The babassu oil constitutes $66 \%$ of kernel weight, and its composition is mainly saturated ( $83 \%$ of the grease composition) which makes it an excellent alternative for biodiesel production. In fact, in Brazil there is a range of oilseed crops that can be used in biodiesel production; currently almost all manufacturing process uses soybean oil as the main raw material. However, some oilseeds, especially soybean oil, run directly into the food industry market, besides extensive area must be used to afford good production. In this sense, non edible crops, as the babassu oil which presents annual productivity and a good yield per hectare, became an excellent alternative [4]. 
This work proposed ethanolysis of babassu oil employing the alkaline hydroxides most commercially used, the sodium and potassium hydroxides, which were evaluated independently. The aim was to study the process performance aided by statistical methodology proposed by Genichi Taguchi [5] to obtain robust processes, i.e., processes with low variation due to uncontrolled variables. So, the homogeneous alkaline transesterification reaction was evaluated in the presence of side reactions - the saponification and hydrolysis, which are inherent to the use of these hydroxides to generate the effective catalysts [6].

\section{Methodology}

The Taguchi designs with orthogonal arrays were performed according to the factors and levels presented in Table 1 to the conventional agitation and in Table 2 to the ultrasound method.

Table 1. Levels and controllable factors used in babassu oil ethanolysis reaction with conventional agitation.

\begin{tabular}{lccc}
\hline \multicolumn{1}{c}{ Factor } & Units & \multicolumn{2}{c}{ Levels } \\
& & 200 & 2 \\
\hline Turbulence & $\mathrm{rpm}$ & 30 & 400 \\
Temperature & ${ }^{\circ} \mathrm{C}$ & $4: 1$ & 60 \\
Molar ratio ethanol/oil & $\mathrm{Mol}$ & 0.5 & $6: 1$ \\
Hydroxide/oil ratio & $\%$ by weight & 30 & 1.0 \\
Reaction time & min & $\mathrm{NaOH}$ & 60 \\
Hydroxide type & & & $\mathrm{KOH}$ \\
\hline
\end{tabular}

Table 2. Levels and controllable factors used to babassu oil ethanolysis reaction with ultrasound agitation.

\begin{tabular}{lccc}
\hline \multicolumn{1}{c}{ Factor } & Units & \multicolumn{2}{c}{ Levels } \\
& & $3: 1$ & 2 \\
\hline Molar ratio ethanol/oil & Mol & 0.5 & $6: 1$ \\
Hydroxide/oil ratio & \% by weight & 10 & 1.0 \\
Reaction time & min & $\mathrm{NaOH}$ & 20 \\
Hydroxide type & & $\mathrm{KOH}$ \\
\hline
\end{tabular}

\subsection{Materials}

Refined babassu oil was kindly provided by the company COGNIS Brazil Ltda.; NaOH $(99 \%), \mathrm{KOH}(85 \%), \mathrm{Na}_{2} \mathrm{SO}_{4}$, anhydrous ethanol (99.8\%) and hexane were obtained in analytical grade and used as received. The properties of the babassu oil in terms of fat acid composition was not determined and it was used the values available in the literature [7].

\subsection{Procedure to the conventional transesterification reaction}

Catalyst and anhydrous ethanol were premixed at $40{ }^{\circ} \mathrm{C}$ under magnetic stirring for $20 \mathrm{~min}$ or until complete dissolution. Then, the solution of the corresponding ethoxide was added to $70 \mathrm{~g}$ of refined babassu oil, previously heated at the same temperature. The reactions were performed in a jacketed glass reactor of $300 \mathrm{~mL}$ with reflux condenser; the temperature control was performed by at hermostatic bath. Mechanical stirring was performed with a mechanical stirrer and glass double curved blades; rotation control was done by mixer brand IKA RW20-digital model. After the predetermined time for each reaction, the reaction mixture was transferred to a $\mathrm{v}$ essel to ensure phase separation between ethyl esters and glycerin. 


\subsection{Procedure to the transesterification reaction with ultrasound}

In a $125 \mathrm{~mL}$ Erlenmeyer flask, it was added $22 \mathrm{~g}$ of refined babassu oil. In parallel, appropriate amounts of anhydrous ethyl alcohol and sodium or potassium hydroxides were mixed until complete dissolution in a glass flask equipped with reflux condenser, under moderate magnetic stirring for $20 \mathrm{~min}$ at $40{ }^{\circ} \mathrm{C}$. In the sequence, the solution containing the corresponding ethoxide catalyst was added to the flask containing the oil preheated to $30{ }^{\circ} \mathrm{C}$. The tub of ultrasonic bath was filled with $300 \mathrm{~mL}$ of distilled water and then the flask containing the reactants was placed inside. The temperature was maintained in $30{ }^{\circ} \mathrm{C}$ and the flask was not sealed, considering that at this temperature the evaporation of ethanol is negligible. The position inside the tub and height of the flask were standardized with markers, in order to use always the same tridimensional position. The equipment was set to operate at $600 \mathrm{~W}$ and $20 \mathrm{kHz}$.

\subsection{Purification process and conversion evaluation}

After phase separation and removal of glycerin produced (lower phase), $200 \mathrm{~mL}$ of hexane were added to the upper phase containing the non reacted intermediates and ethyl esters. This promotes new glycerin phase separation. After collecting this new phase, a new step involving several washings with 0.1 mol. $\mathrm{L}^{-1}$ solution of $\mathrm{HCl}$ were done, in order to reach neutral $\mathrm{pH}$. After rinsing, this phase was dried with approximately $0.5 \mathrm{~g}$ of anhydrous $\mathrm{Na}_{2} \mathrm{SO}_{4}$ to remove remaining water, followed by vacuum filtration and, finally, residual alcohol and hexane were evaporated by a rotary evaporator at $72{ }^{\circ} \mathrm{C}$ during 20 min under atmospheric pressure.

The conversion into ethyl esters was evaluated by NMR in a Mercury $300 \mathrm{MHz}-$ Varian spectrometer, with $5 \mathrm{~mm}$ glass tubes, using $\mathrm{CDCl}_{3}$ as solvent and $0.3 \%$ TMS as internal standard. The calculations involving the conversion of esters were determined using the formula proposed by Garcia [8]. This methodology basically consisted in the identification, by ${ }^{1} \mathrm{H}$ NMR, of molecules that present peaks in the region of 4.05 to $4.35 \mathrm{ppm}$ during a transesterification reaction.

\section{Results}

\subsection{Evaluation of controllable variables as a function of the process noises}

According to the methodology proposed by Taguchi [5], the signal to noise ratio $(\mathrm{S} / \mathrm{N})$ can, in this case, be interpreted as conversion into ethyl esters in the presence of noise (uncontrollable) factors. Figure 1 shows the effects of controllable factors in the signal to noise ratio, measured independently, i.e. without considering interactions between these factors, this figure was produced by the software Minitab ${ }^{\circledR}$, that was used to perform the statistical analysis.

\subsection{Molar ratio ethanol / babassu oil in the conventional procedure}

The data showed that among all the factors, taken individually, the ethanol / oil ratio was the most important factor in conversions into ethyl esters. Due to the existence of a dynamic equilibrium between reactants and products, it is expected that excess alcohol increases the conversion to esters. In this sense, the results show that ethanolysis of babassu oil behaves similarly to the results already reported to other vegetable oils.

One of the advantages of applying statistical designs on the experiments is the evaluation of interactions between the factors; sometimes these interactions may be more important than the controlled variables. As stated, this factor presents a significant interaction with the reaction time, as it could be demonstrated by the interactions analysis, showing that regardless of the 
time adopted (30 or $60 \mathrm{~min}$ ), the conversions are higher when used the molar ratio $6: 1$ of ethanol / oil.

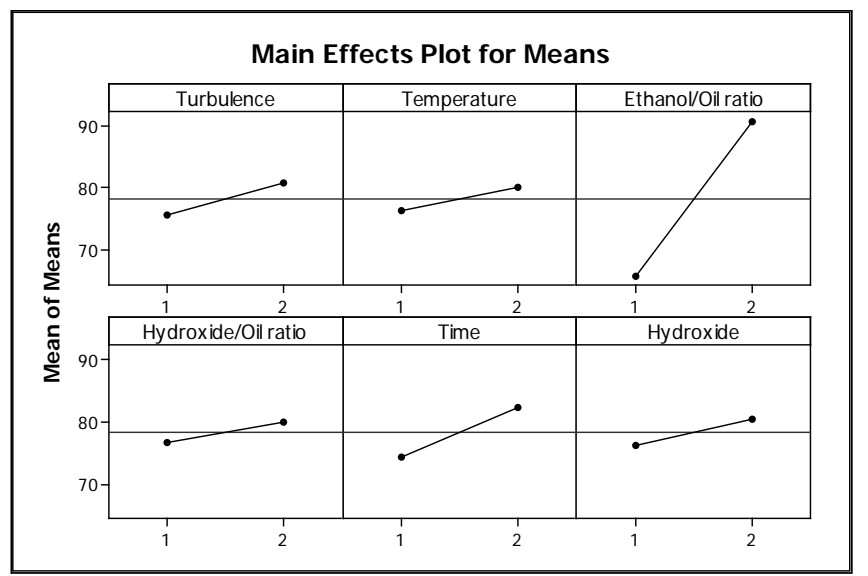

* 1 and 2 correspond to low and high levels respectively

Fig. 1. Effects of controllable factors in the average conversion to ethyl esters as a function of noise in the conventional procedure

\subsection{Effect of temperature}

When temperature is evaluated individually its effect is modest, as it can be observed in Figure 1. However, considering the interactions, best conversions are obtained with temperatures set at low level, i.e., $30{ }^{\circ} \mathrm{C}$ (Figure 2a). Likewise, the turbulence generated by mechanical agitation at $400 \mathrm{rpm}$ promotes best conversion into ethyl esters with temperature of $30{ }^{\circ} \mathrm{C}$ (Figure 2b). This peculiar behavior can be explained by hydrolysis and saponification reactions, enhanced by higher temperatures, which promote the consumption of the catalyst reducing the yield of conversions [6].

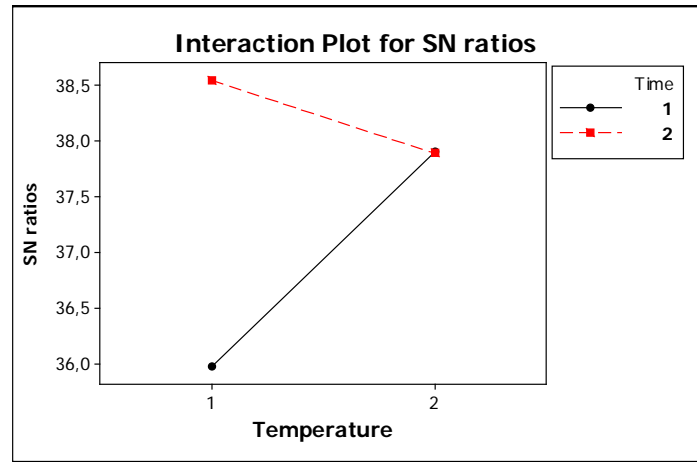

(a)

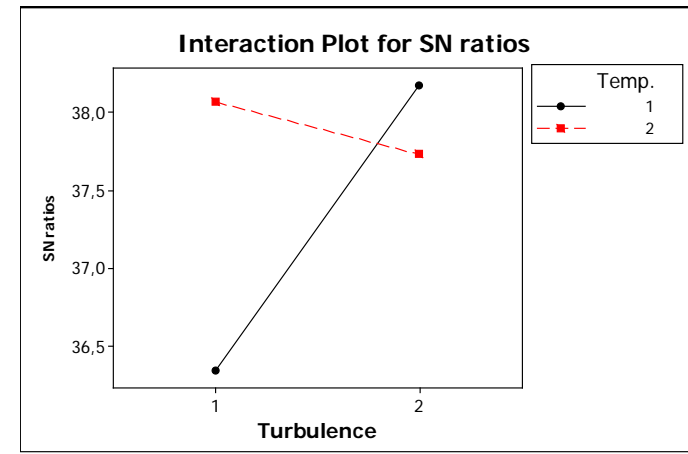

(b)

Fig. 2. Interaction of (a) temperature/time and (b) temperature/turbulence on the signal to noise ratio in the conventional procedure

\subsection{Effect of amount and type of hydroxide}

In most industrial processes the catalyst is expensive when compared to reagents and adds additional costs for its removal from the final product. The effective catalyst in a homogeneous transesterification reaction is the anion formed from the reaction between basic hydroxide and the alcohol. However, for practical and industrial purposes, the percentage ratio by weight of the hydroxide to vegetable oil is commonly used to describe the effect of the catalyst involved.

It was used $1 \%(\mathrm{wt})$ of both hydroxides, what is equivalent to $0.0125 \mathrm{~mol}$ of $\mathrm{KOH}$ and $0.0175 \mathrm{~mol}$ of $\mathrm{NaOH}$. So, regarding the type of hydroxide, the data show that the use of $\mathrm{KOH}$ 
is desirable, since lower molar quantities promoted better conversion into ethyl esters. Furthermore, the purification procedures were significantly facilitated when using potassium hydroxide, this experimental finding supports the conclusion that the generation of soap with $\mathrm{KOH}$ is lower.

Regarding the amount of hydroxide, this study showed that the use of $1 \%$ by weight of it in relation to the mass of babassu oil leads to better results.

\subsection{Effect of turbulence}

The literature highlights the importance of agitation during the early stages of alkaline transesterification reactions, because during the initial stages, the mass transfer is limited $[9,10]$. The results suggested that the turbulence levels adopted in this study are sufficient for good conversions into ethyl esters, i.e., higher values of mechanical agitation than $400 \mathrm{rpm}$ do not improve the yield of conversion.

Besides interaction with the temperature, this factor also had another significant interaction with the amount of hydroxide used, showing that the best conversions are obtained when these factors are set at higher level, i.e., mechanical agitation of $400 \mathrm{rpm}$ and $1 \%$ hydroxide (Figure 3). The behavior exhibited by this interaction can be explained by an improvement in the conditions of mass transfer with more turbulence and also due to increase on availability of catalysts for the reaction with the use of $1 \%$ hydroxide in relation to initial mass of oil babassu.

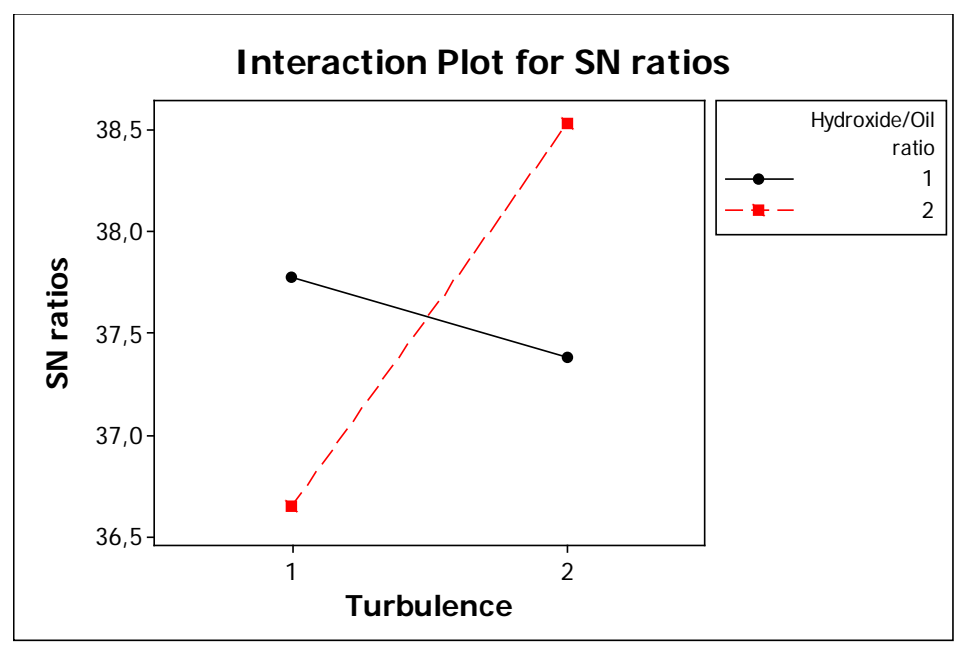

Fig. 3. Interaction plot between turbulence and \% by weight ratio hydroxide / babassu oil in the conventional procedure

\subsection{Effect of Time}

The reaction time showed to be a dependent controllable factor, and with optimization of no other factors, the data showed that satisfactory conversions were reached within $60 \mathrm{~m}$ in of reaction.

Thus the technological model obtained with this methodology was adjusted to the temperature at $30{ }^{\circ} \mathrm{C}, 400 \mathrm{rpm}$ of mechanical agitation, using $1 \%$ potassium hydroxide during $60 \mathrm{~min}$ of reaction. The conversion into ethyl esters obtained were above $99 \%$, determined by ${ }^{1} \mathrm{H}$ NMR, and the weight of esters recovered after the purification procedures was $94.59 \%$ compared to the initial mass of this product. 


\subsection{Mathematical model}

The evaluation of controllable factors to babassu oil ethanolysis reaction as a function of the noises was useful in determining the influence of the main variables in the conversion to ethyl esters, as can be seen in the Figure 1 and in the discussion about the effect of the temperature. After that, new experimental design was employed with the two main variables and the response surface methodology (RSM) was applied, with the development of new $2^{2}$ complete factorial design with axial points, in order to identify the optimum conditions of babassu oil ethanolysis.

The mathematical model proposed to describe babassu oil ethanolysis is shown in Equation (1). The quadratic coefficients of correlation show that the model can explain $96.21 \%$ of the variability in the response and further simulations with this equation are able to predict 80.47 $\%$ of the results.

$\% \mathrm{CEE}=54.37-1.836 \theta+3.020 \mathrm{r}+0.021 \theta^{2}-0.025 r^{2}-0.007(\theta \times r)$

Where \% $\mathrm{CEE}$ is the ethyl esters conversion percentage, $\theta$ is the temperature and $\mathrm{r}$ the ethanol/Oil ratio

Figure 4 was generated with the software Minitab ${ }^{\circledR}$ from Eq. (1) and show the best fit to a babassu oil ethanolysis. As can be seen, with temperatures below $40{ }^{\circ} \mathrm{C}$ there is a $\mathrm{n}$ arrow range in which the conversion into ethyl esters is higher, reaching the highest value in temperature around $30^{\circ} \mathrm{C}$ and ethanol/oil ratio around $6: 1$ (60 in the figure).

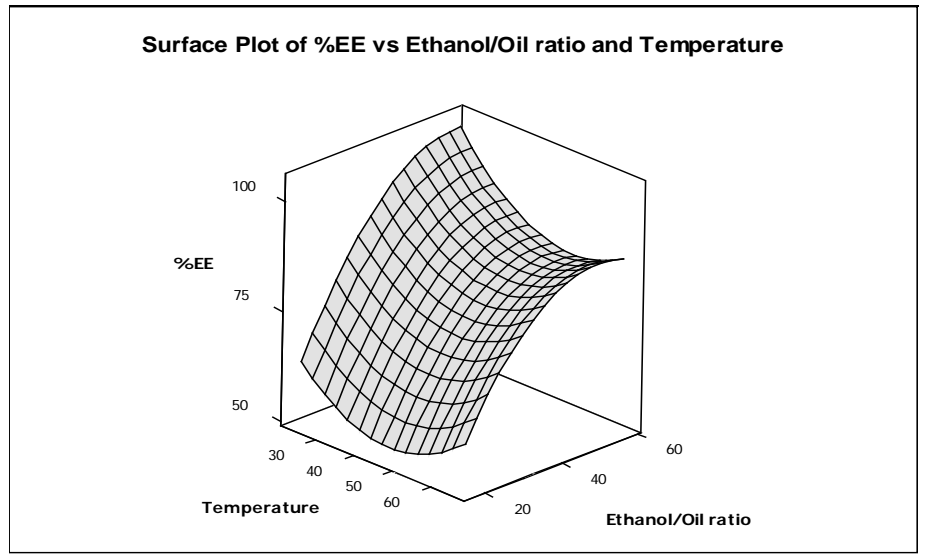

Fig. 4. Response surface to ethyl esters conversions as a function of the ethanol/oil ratio and temperature in the conventional procedure

\subsection{The effect of the use of ultrasound}

As it can be seen in de Figure 5, since there are no crossings between the effect lines of the variables, does not exist interactions of relevance to this system, thus the interpretation of controllable factors can be made directly.

In the Figure 6 it can be observed that the ratio ethanol/oil was also the most important factor involved in ethanolysis under ultrasound, showing that both processes, traditional agitation and sonication, are primarily dependent on this factor; no matter how intense are the physical conditions to which the medium is submitted. Similar results were reported in literature to the ethanolysis coconut oil [11]. 
Figure 6 also shows that the ratio hydroxide/oil was relevant to the sonolysis of babassu oil, the degree of conversion significantly responded to the variation of levels. The best conversions are obtained when employing the mass ratio of $1.0 \%$ (wt) of catalyst of babassu oil. This result was expected because the greater availability of catalyst greater the generation of the active component of the reaction, in this case, the ethoxide catalyst and, due to the phenomena of sonoluminescence and cavitation noise, which are responsible for creating unique conditions for transfer mass, the greater amount of this active component had its effect enhanced [12].

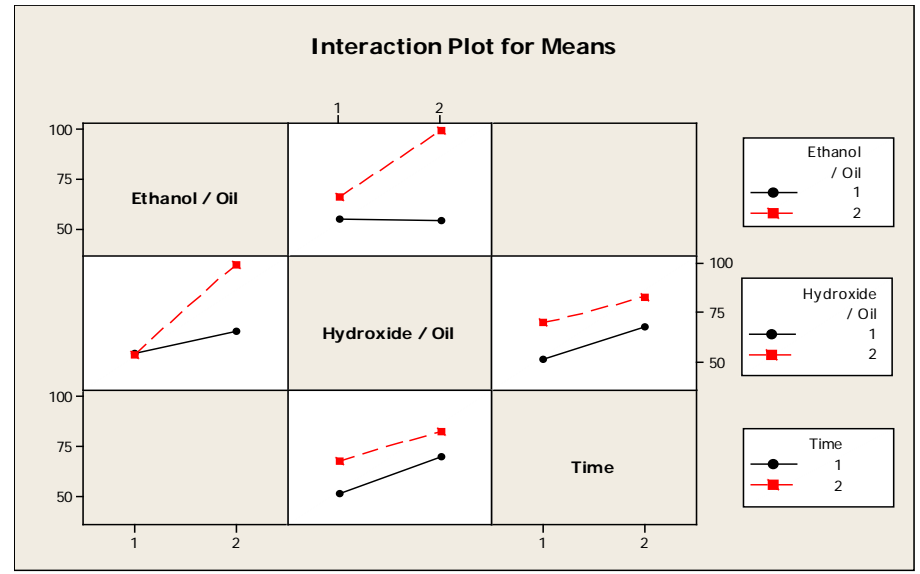

Fig. 5. Interaction plot on the signal to noise ratio for studied variables in the sonication procedure

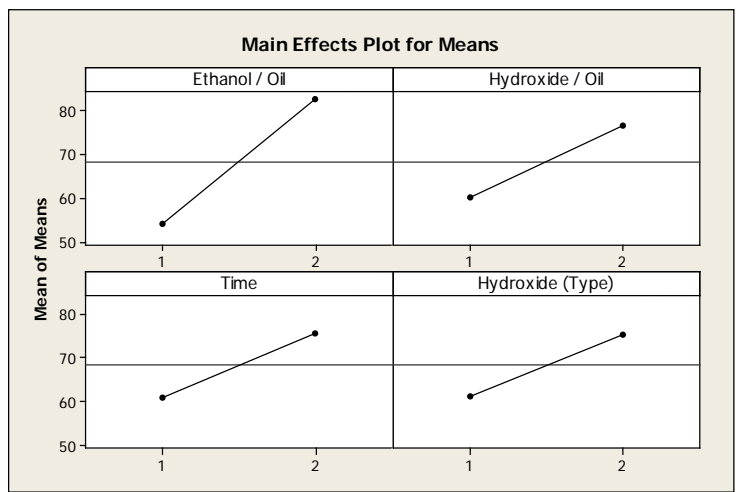

Fig. 6. Effects of controllable factors in the average conversion to ethyl esters as a function of noise in the sonication method

When compared to the traditional method, the time factor of sonication was considerably reduced and conversions above $99 \%$ were obtained in 10 min, meaning that despite the significance showed for this variable in Figure 6, there is no practical reason to use the upper level of time. The important reduction in time compared to traditional methodology, can be explained by the intense mass transfer promoted by cavitation noise, implying a reduction of time intervals spent on the diffusion of reagents.

\section{Conclusions}

The refined babassu oil has shown excellent qualities as a raw material. Even in ethanolysis reaction this oil presented a similar behavior to reactions that used methanol showed in the literature $[4,13]$. The experiments showed that the correct tuning in the process variables is able to promote higher conversions into ethyl esters. 
Among all the controllable factors evaluated, the ratio ethanol/oil decisively influenced the conversions into ethyl esters; highest conversions are achieved at the stoichiometric ratio of 6:1. The temperature showed a peculiar behavior, pointing out that the best conversions are obtained at $30{ }^{\circ} \mathrm{C}$. Other important conclusion is about the amount of hydroxide employed that suggests that a rate around $1.0 \%$ is sufficient to obtain good conversions. $\mathrm{KOH}$ proved to be preferable if compared to $\mathrm{NaOH}$, since better conversions were obtained and purification steps were easier.

The sonolysis of babassu oil showed considerable gain in time with respect to classical transesterification, principally during the stages of phase separation, which were substantially facilitated. Similar results were obtained for the preparation of biodiesel by the transesterification of coconut oil [11]. The remarkable results obtained with the reaction time can be explained by intense mass transfer afforded by the unique conditions generated by cavitation noise. In addition, possible reductions in the concentrations of mono- and diglycerides during the reaction may explain the reduction in the time for phase separation. Thus, the method presents itself as a potential technological route of production of biodiesel, capable of meeting high demands in short periods of time. Adjustments related to the type of ultrasonic reactor (or transducer) and in the process of vegetable oils sonolysis can lead to an excellent alternative for biodiesel production, with energy costs that may be less than the expenses involved with the traditional method of mechanical agitation.

\section{References}

[1] Pinto, A. C., Guarieiro, L. L. N., Rezende, M. J. C., Ribeiro, N. M., Torres, E. A., Lopes, W. A., Pereira, P. A. P.; de Andrade; J. Braz. Chem. Soc 2005, v. 16, p. 1313.

[2] Knothe, G., Dunn, R. O., Biofuels Derived from Vegetable Oils and Fats. In Oleochemical Manufacture and Applications. Academic Press: Sheffield, U.K., 2001.

[3] Dorado, M. P., Ballesteros, E., Almeida, J. A.; Schellet, C., Lohrlein, H. P., Krause, R., Trans ASAE 2005, v. 45, p. 525.

[4] Lima, J. R.; Silva, R. B.; Silva, C. C. M.; Santos, L. S. S.; Santos JR., J. R.; Moura, E. M.; de Moura, C. V. R., Química Nova, 2007, v.30, n.3, pp. 600-603.

[5] Taguchi, G. Introduction to Quality Engineering: Designing Quality into Products and Processes, White Plains, NY. Kraus International Publications, 1986.

[6] Schuchardt, U.; Sercheli, R.; Vargas, R. M. Transesterification of vegetable oils: a review. J. Braz. Chem. Soc., 1998, v.9, pp. 199-210.

[7] Gunstone, F. D.; Harwood, J. L.; Padley, P. The Lipid Handbook 2. ed. London: Chapman and Hall, 1994.

[8] Garcia, C. M., Master Dissertation - State Univ. of Campinas, UNICAMP, Brazil, 2006.

[9] Noureddini, H.; Zhu, D. J. Am. Oil Chem. Soc., 1997, v.74.

[10]Freedman, B.; Butterfield, R. O.; Pryde, E. H. Transesterification kinetics of soybean oil, J. Am. Oil Chem. Soc., 1986, v. 63.

[11]Kumar, D., Kumar, G., Singh, P. C. P., Ultrasonics Sonochemistry, 2010, v.17, pp. 555559.

[12] Stavarache, C.; Vinatoru, M.; Maeda, Y. Ultrasonic versus silent methylation of vegetable oils, Ultrasonics Sonochemistry, 2006, v.13, pp. 401-407.

[13]Nogueira, Jr., C. A. F.; Filipe, X.; Fernandes, F. A. N., Santiago, R. S. and Sant'Ana, H. B., J. Chem. Eng. Data 2010, v. 55, pp. 5305-5310. 